\title{
Theoretical Analysis and Improvement of Output Voltage for Resonant Cockcroft-Walton Circuit
}

\author{
Masataka Minami*a) Member, Takumi Yasuda,*** Student Member \\ Jun-ichi Itoh ${ }^{* *}$ Senior Member
}

(Manuscript received March 4, 2020, revised July 26, 2020)

J-STAGE Advance published date : Oct. 1, 2020

\begin{abstract}
The Cockcroft-Walton (CW) circuit is used in high-voltage low-current apparatuses, such as electron beam irradiation devices and insulation testing devices. The parasitic capacitance of the $\mathrm{CW}$ circuit is not considered in the design of a high voltage resonant converter. In the past, it has been clarified that the equivalent capacitor of the CW circuit can be utilized as a resonant capacitor to boost the output voltage. This study derives a theoretical quality factor and an output voltage of a resonant $\mathrm{CW}$ circuit, considering the equivalent capacitance and the equivalent conductance. Furthermore, it is revealed that parallel capacitors can improve the quality factor and the output voltage of the CW circuit. Experimental results obtained from the 5-stage resonant $\mathrm{CW}$ circuit verify the validity of the theoretical analysis.
\end{abstract}

Keywords: Cockcroft-Walton circuit, $L C$ resonance, quality factor, boost ratio

\section{Introduction}

The Cockcroft-Walton $(\mathrm{CW})$ circuit $^{(1)}$ has been widely used in many high-voltage/low-current industrial applications where a low-cost and compact system is required such as electron beam irradiation system, insulation testing devices, and so on ${ }^{(2)-(4)}$. The $\mathrm{CW}$ circuit has advantages of the simple construction and no active switches. The ideal output voltage of the unloaded $N$-stage $\mathrm{CW}$ circuit is $2 N$ times of the amplitude of the input voltage $V_{\text {in }}{ }^{(1)}$. However, a practical CW circuit has an output voltage drop due to the diode junction capacitors ${ }^{(5)(6)}$. In order to overcome the output voltage drop, several studies have been made such as improved constructions ${ }^{(5)(7)}$ and an optimization of parameters ${ }^{(8)}$. The output voltage of the $\mathrm{CW}$ circuit becomes close to the ideal output voltage $2 N V_{\text {in }}$ thanks to these techniques. However, the output voltage of the $\mathrm{CW}$ circuit adopted the previous methods never exceeds the ideal output voltage $2 N V_{\text {in }}$ since the previous methods tried to eliminate the influence of the diode junction capacitors.

Generally, isolated high step-up DC-DC converters need to adopt a transformer with large turn ratio. Therefore, parasitic components of the transformer are not ignored for the theoretical analyses. In these converters, a leakage inductor and a winding capacitor in the transformer construct the parallel resonant circuit and boost the output voltage ${ }^{(3)(9)(10)}$. On the other hand, the $\mathrm{CW}$ circuit adopted for use in the high step-up DC-DC converters is assumed as a resistor ${ }^{(11)}$ or an ideal voltage source ${ }^{(12)}$. Because the equivalent capacitance

a) Correspondence to: Masataka Minami. Email: minami@kobekosen.ac.jp

* Kobe City College of Technology

8-3, Gakuenhigashi, Nishi-ku, Kobe 651-2194, Japan

${ }^{* *}$ Nagaoka University of Technology

1603-1, Kamitomioka, Nagaoka, Niigata 940-2188, Japan of the CW circuit is smaller than the winding capacitance of the transformer, it is ignored for the analysis of the converter. Recently, the operating frequency of the converter tends to be higher thanks to the wide-band-gap semiconductors ${ }^{(13)-(15)}$. The high-frequency operation helps to reduce the number of turns and the parasitic components of the transformer. As a result, the equivalent capacitance of the $\mathrm{CW}$ circuit cannot be ignored in the high-frequency and high step-up resonant DC-DC converters. Therefore, we revealed that the equivalent capacitor of the $\mathrm{CW}$ circuit can be utilized as a resonant capacitor for improvement of the output voltage ${ }^{(16)}$. The previous paper ${ }^{(16)}$ theoretically developed the equivalent circuit by approximating only capacitors without resistors.

This paper derives a theoretical output voltage of a resonant $\mathrm{CW}$ circuit considering the equivalent capacitor and the equivalent conductor. It is clarified that the additional parallel capacitors improve the output voltage of the resonant $\mathrm{CW}$ circuit. Finally, the experimental results reveal the validity of the theoretical analysis. Section 2 describes an operating principle and a theoretical analysis of the resonant CW circuit. Particularly, an output voltage of the proposed resonant $\mathrm{CW}$ circuit is derived using a quality factor. An experimental verification is undergone in Section 3. The theoretical output voltage is compared with the experimental one in Section 4.

\section{Proposed $N$-stage Resonant $\mathrm{CW}$ Circuit}

Fig. 1 depicts a proposed $N$-stage resonant $\mathrm{CW}$ circuit with parallel capacitors. This section describes a principle and operation states of the proposed $N$-stage resonant $\mathrm{CW}$ circuit. Besides, a quality factor and the output voltage are theoretically derived.

2.1 Principle The $N$-stage resonant $\mathrm{CW}$ circuit involves an inductor $L$ in series with the input side and parallel capacitors $C_{\mathrm{r}}$ in parallel with each diode $D_{1}, D_{2}, \ldots$, and $D_{2 N}$. The inductor $L$ represents the circuit parasitic inductor, 


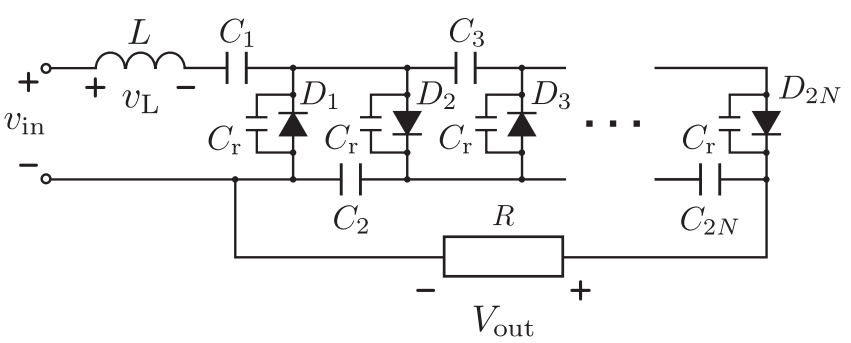

Fig. 1. Proposed $N$-stage resonant $\mathrm{CW}$ circuit with parallel capacitors

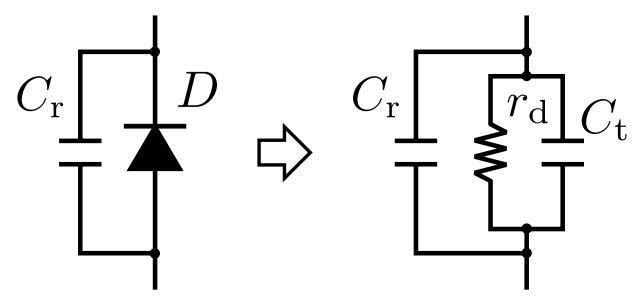

Fig. 2. Equivalent circuit of diode and external parallel capacitor

which is the leakage inductor of the transformer or an additional inductor for the $L C$ resonance. As Section 1 mentioned, the conventional $\mathrm{CW}$ circuit is generally utilized in the apparatuses that require high-voltage and low-current. Therefore, the ON-period of the diodes is short because of low load current. As a result, the diodes of the conventional $\mathrm{CW}$ circuit are regarded as the junction capacitor $C_{\mathrm{t}}$ in almost period $^{(5)(17)}$.

Fig. 2 shows an equivalent circuit of the diode in the OFFstate $^{(18)}$ and the external parallel capacitor. The diode in the OFF-state acts as a resistor $r_{\mathrm{d}}$ and the junction capacitor $C_{\mathrm{t}}$. When the capacitors $C_{1}, C_{2}, \ldots, C_{2 N}$ in Fig. 1 is much larger than the sum of the junction capacitor $C_{\mathrm{t}}$ and the inserted parallel capacitor $C_{\mathrm{r}}$, an equivalent circuit of the proposed resonant CW circuit is regarded as only parallel connection of the junction capacitors $C_{\mathrm{t}}$ and the parallel capacitors $C_{\mathrm{r}}$. In other words, the equivalent capacitor $C_{\text {all }}$ of the proposed resonant $\mathrm{CW}$ circuit in Fig. 1 is approximately equal to the sum of the junction capacitors $C_{\mathrm{t}}$ and the parallel capacitors $C_{\mathrm{r}}$, whose capacitance is $2 N\left(C_{\mathrm{t}}+C_{\mathrm{r}}\right)$. Therefore, it is considered that the output voltage of the proposed resonant $\mathrm{CW}$ circuit can be increased by an $L C$ resonance between the inductor $L$ and the equivalent capacitor $C_{\text {all }}$.

Actually, the diode junction resistor ${ }^{(18)}$ cannot be ignored for the proposed resonant $\mathrm{CW}$ circuit. In the theoretical analysis, the junction resistors in the proposed resonant $\mathrm{CW}$ circuit are considered for derivation of the quality factor and the output voltage in this paper.

2.2 Operation Modes Fig. 3 illustrates the operation waveforms of the proposed 2-stage resonant $\mathrm{CW}$ circuit operating at around the resonant frequency between the inductor $L$ and the equivalent capacitor $C_{\text {all }}$. Moreover, Fig. 4 draws the operation modes in one period of the proposed 2-stage resonant CW circuit. Here, Label $i_{\mathrm{D} n}(1 \leq n \leq 4)$ for the current waveform means the current flowing from top to bottom of the diode $D_{n}$. The input voltage $v_{\text {in }}$ is square-wave with amplitude $10 \mathrm{~V}$ and the load $R$ is set to $100 \mathrm{k} \Omega$.

At the first of the positive/negative-half cycle: Mode 1 and

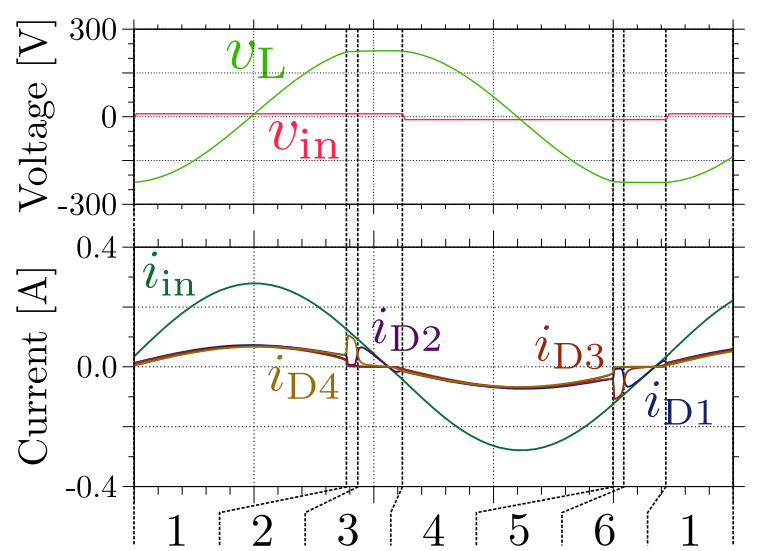

Fig. 3. Voltage and current waveforms of proposed 2stage resonant $\mathrm{CW}$ circuit at resonant frequency by numerical simulation

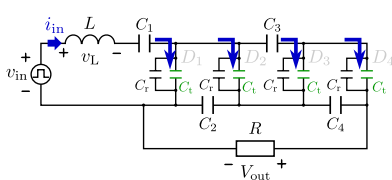

(a) Mode 1

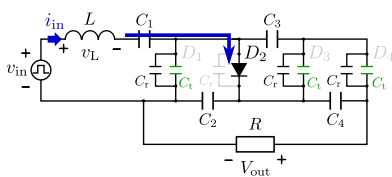

(c) Mode 3

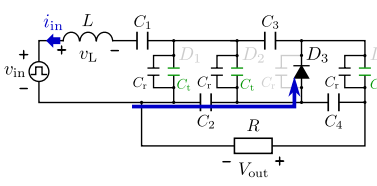

(e) Mode 5

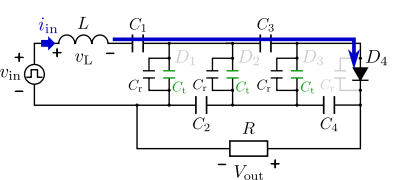

(b) Mode 2

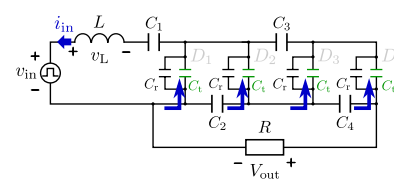

(d) Mode 4

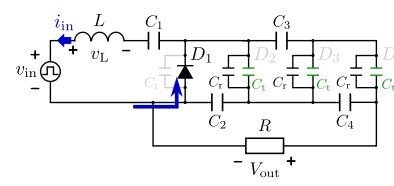

(f) Mode 6
Fig. 4. Operation modes of proposed 2-stage resonant CW circuit

Mode 4, all diodes are OFF and they act as the junction capacitors $C_{\mathrm{t}}$. The inductor $L$ resonates with the junction capacitors $C_{\mathrm{t}}$ and the parallel capacitors $C_{\mathrm{r}}$ in the periods. As a result, the voltage of the power supply and the inductor: $\left(v_{\text {in }}-v_{\mathrm{L}}\right)$ is boosted. Accordingly, the output voltage $V_{\text {out }}$ increases. In the modes, the inductor $L$ and the equivalent capacitor $C_{\text {all }}$ act as a parallel resonant circuit $^{(19)(20)}$.

On the other hand, at the second and third of the positive/negative-half cycle: Mode 2-3 and Mode 5-6, $D_{4}$ $D_{2}$ and $D_{3}-D_{1}$ respectively conduct. The conducting sequences are $D_{4}-D_{2}$ in the positive-half cycle and $D_{3}-D_{1}$ in the negative-half cycle of the input voltage $v_{\text {in }}$ respectively as shown in Mode 2-3 and Mode 5-6. The conduction sequences are also observed in the conventional $\mathrm{CW}$ circuit $^{(3)(21)}$. As shown in Fig. 3, the inductor voltage $v_{\mathrm{L}}$ is constant in Mode $2,3,5$, and 6 . In the mode, the inductor voltage $v_{\mathrm{L}}$ is clamped to the capacitor voltage. These modes begin after the voltage $\left(v_{\text {in }}-v_{\mathrm{L}}\right)$ reaches peak value. Therefore, the voltage at each capacitor is approximately equal to the maximum voltage in Mode 1 and Mode 4. The theoretical output voltage can be calculated with the maximum voltage in Mode 1 and Mode 4.

The operation modes of the proposed $N$-stage resonant $\mathrm{CW}$ circuit are almost the same as the proposed 2-stage resonant CW circuit. 


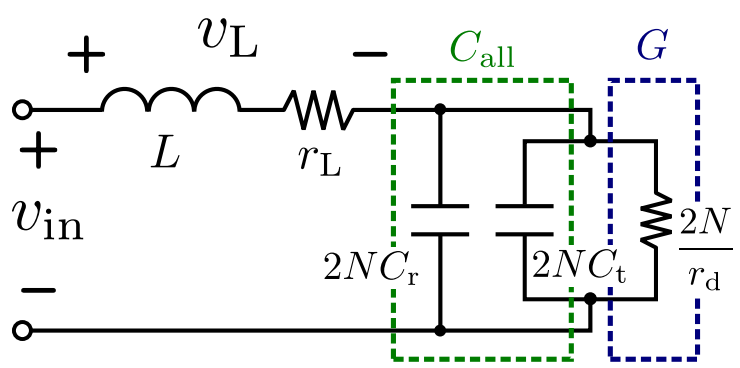

Fig. 5. Equivalent circuit of $N$-stage resonant $\mathrm{CW}$ circuit considering equivalent circuit of diodes

2.3 Quality Factor The proposed resonant CW circuit behaves as a parallel resonant converter composed of the inductor $L$, the junction capacitor $C_{\mathrm{t}}$, and the parallel capacitor $C_{\mathrm{r}}$ as mentioned above. Therefore, a higher quality factor of the converter leads to a higher output voltage ${ }^{(19)(20)}$.

Here, the capacitors $C_{1}, C_{2}, \ldots, C_{2 N}$ are assumed to be much larger than the parallel capacitors $C_{\mathrm{r}}$. Hence, the equivalent circuit of Fig. 1 is drawn in Fig. 5, which consists of only the inductor $L$, the diodes $D_{1}, D_{2}, \ldots, D_{2 N}$, and the parallel capacitors $C_{\mathrm{r}}$. In Fig. 5 , the equivalent circuit of the diodes is assumed as the parallel connection of the junction capacitor $C_{\mathrm{t}}$ and the junction resistor $r_{\mathrm{d}}{ }^{(18)}$.

First, the quality factor $Q$ is defined as

$$
Q=\frac{\omega_{0}}{2}\left|\frac{z^{\prime}\left(\omega_{0}\right)}{z\left(\omega_{0}\right)}\right|
$$

where $\omega$ and $z(\omega)$ denote an angular frequency of the input voltage $v_{\text {in }}$ and the impedance of Fig. 5, the apostrophe $\left(^{\prime}\right)$ is defined as $d / d \omega$, and $\omega_{0}$ means the resonant angular frequency from $\operatorname{Im}\left[z\left(\omega_{0}\right)\right]=0{ }^{(22)}$. Then, the impedance of Fig. 5 is expressed as

$$
z\left(\omega_{0}\right)=r_{\mathrm{L}}+\mathrm{j} \omega_{0} L+\frac{1}{G+\mathrm{j} \omega_{0} C_{\mathrm{all}}}
$$

and

$$
z^{\prime}\left(\omega_{0}\right)=\mathrm{j} L-\frac{\mathrm{j} C_{\mathrm{all}}}{\left(G+\mathrm{j} \omega_{0} C_{\mathrm{all}}\right)^{2}},
$$

where $r_{\mathrm{L}}$ means the internal resistor of the inductor $L$. In (2), $G$ and $C_{\text {all }}$ are defined as $G=2 N / r_{\mathrm{d}}$ and $C_{\text {all }}=2 N\left(C_{\mathrm{r}}+C_{\mathrm{t}}\right)$. Therefore, the quality factor $Q$ is derived as

$$
Q=\frac{\omega_{0}}{2}\left|\frac{\mathrm{j} L-\frac{\mathrm{j} C_{\mathrm{all}}}{\left(G+\mathrm{j} \omega_{0} C_{\mathrm{all}}\right)^{2}}}{r_{\mathrm{L}}+\mathrm{j} \omega_{0} L+\frac{1}{G+\mathrm{j} \omega_{0} C_{\mathrm{all}}}}\right|
$$

On the other hand, the resonant angular frequency $\omega_{0}$ calculated from $\operatorname{Im}\left[z\left(\omega_{0}\right)\right]=0$ is obtained as

$$
\omega_{0}^{2}=\frac{1}{L C_{\text {all }}}-\left(\frac{G}{C_{\text {all }}}\right)^{2}
$$

(4) and (5) lead the quality factor $Q$ as

$$
Q=\frac{\omega_{0}^{2}\left(L C_{\mathrm{all}}\right)^{3 / 2}}{C_{\mathrm{all}} r_{\mathrm{L}}+L G}
$$

Next subsection derives the theoretical output voltage using the quality factor $Q$ of the proposed resonant $\mathrm{CW}$ circuit. And it is clarified that the higher quality factor $Q$ helps to boost the output voltage.
2.4 Theoretical Output Voltage As the proposed resonant $\mathrm{CW}$ circuit is regarded as a conventional $\mathrm{CW}$ circuit whose input voltage is a difference between the input voltage $v_{\text {in }}$ and the inductor voltage $v_{\mathrm{L}}$, where $\left(v_{\text {in }}-v_{\mathrm{L}}\right)$, the theoretical output voltage $V_{\text {out,the }}$ can be derived with the conventional calculation method ${ }^{(5)}$.

At first, the voltage $\left(v_{\text {in }}-v_{\mathrm{L}}\right)$ from Fig. 5 is expressed as

$$
v_{\text {in }}-v_{\mathrm{L}}=\frac{\frac{1}{G+\mathrm{j} \omega_{0} C_{\text {all }}}}{r_{\mathrm{L}}+\mathrm{j} \omega_{0} L+\frac{1}{G+\mathrm{j} \omega_{0} C_{\text {all }}}} v_{\text {in }} \ldots \ldots \ldots
$$

As (5) and (6) are assigned to (7), the voltage $\left(v_{\text {in }}-v_{\mathrm{L}}\right)$ is given by

$$
v_{\text {in }}-v_{\mathrm{L}}=\frac{Q}{\omega_{0}^{2}\left(L C_{\text {all }}\right)^{3 / 2}\left(\frac{G}{C_{\text {all }}}+\mathrm{j} \omega_{0}\right)} v_{\text {in }} \ldots \ldots \ldots \ldots
$$

The theoretical output voltage of the conventional $\mathrm{CW}$ circuit is $2 N$ times as large as the amplitude of the input voltage ${ }^{(1)}$. Hence, an ideal output voltage $V_{\text {out,idl }}$ of the proposed $N$-stage resonant $\mathrm{CW}$ circuit without the influence of the diode junction capacitors $C_{\mathrm{t}}$ is obtained in same way as shown in

$$
V_{\text {out }, \text { idl }}=\frac{2 N Q}{\omega_{0}^{2}\left(L C_{\text {all }}\right)^{3 / 2}\left\{\left(\frac{G}{C_{\text {all }}}\right)^{2}+\omega_{0}^{2}\right\}^{1 / 2}} V_{\text {in }}, \cdots \cdots
$$

where $V_{\text {in }}$ denotes the amplitude of the input voltage $v_{\text {in }}$. As (5) is assigned to (9), $V_{\text {out,idl }}$ is deformed as

$$
V_{\text {out }, \text { idl }}=\frac{2 N Q}{\omega_{0}^{2} L C_{\text {all }}} V_{\text {in }} .
$$

It is known that the output voltage drops on account of the diode junction capacitors $C_{\mathrm{t}}{ }^{(5)}$. In the proposed $N$-stage resonant $\mathrm{CW}$ circuit, it is considered that the junction capacitors $C_{\mathrm{t}}$ and the parallel capacitors $C_{\mathrm{r}}$ decrease the output voltage. The effect is expressed by voltage efficiency $F^{(5)}$. Therefore, the theoretical output voltage $V_{\text {out,the }}$ of the $N$-stage resonant $\mathrm{CW}$ circuit is derived as

$$
V_{\text {out,the }}=F V_{\text {out,idl }}=\frac{2 N F Q}{\omega_{0}^{2} L C_{\text {all }}} V_{\text {in }},
$$

where the voltage efficiency $F$ is expressed as

$$
F=\left(\frac{2 C}{N^{2} C_{\text {all }}}\right)^{1 / 2} \tanh \left(\frac{N^{2} C_{\text {all }}}{2 C}\right)^{1 / 2}
$$

where $C$ denotes capacitance of $C_{1}, C_{2}, \ldots, C_{2 N}$. Here, we explain approximately (11). As the $G$ is small enough, (5) is regarded as $\omega_{0}^{2} \approx 1 / L C_{\text {all }}$, that is, $\omega_{0}^{2} L C_{\text {all }} \approx 1$. On the other hand, when $C_{\text {all }}$ is sufficiently smaller than $C, F \approx 1$. From the above, (11) indicates that the output voltage $V_{\text {out,the }}$ is proportional to the quality factor $Q$ only if $C_{\text {all }}$ is sufficiently smaller than $C$. Thus, the equation implies that the improvement of the quality factor $Q$ contributes to boost the output voltage.

As an example, Fig. 6 shows the ideal relationship between 


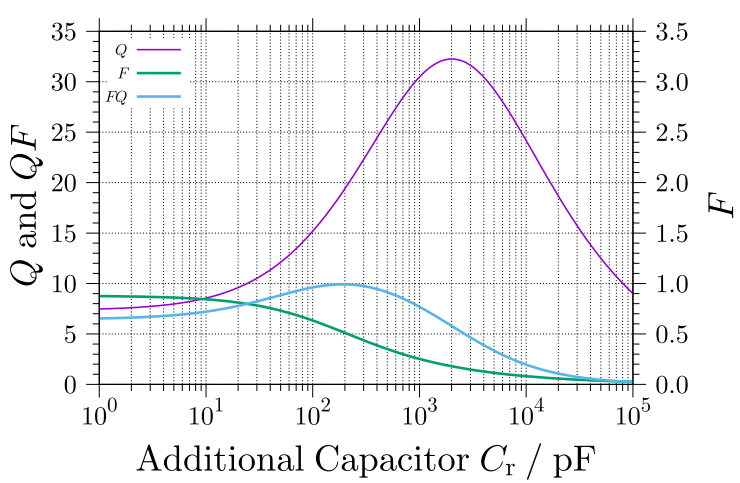

Fig. 6. Ideal relationship between $C_{\mathrm{r}}, Q, F$, and $F Q$ un$\operatorname{der} G=0.2 \mathrm{mS}$

$C_{\mathrm{r}}, Q, F$, and $F Q$ under $G=0.2 \mathrm{mS}^{\dagger}$. From (12) and Fig. 6, when $C_{\mathrm{r}}$ is over $10 \mathrm{pF}$, the voltage efficiency $F$ starts to decrease. It is not possible to approximate $F$ to 1 . Therefore, the output voltage is highly dependent on the $F Q$. As a result, the optimal $C_{\mathrm{r}}$ that maximizes the output voltage exists between $100 \mathrm{pF}$ and $1000 \mathrm{pF}$.

However, it is difficult to consider the junction resistor $r_{\mathrm{d}}$ and the load $R$ separately in the equivalent circuit in Fig. 5 because the $\mathrm{CW}$ circuit is treated as a parallel connection of one conductor $G$ and one capacitor $C_{\text {all }}$. Therefore, the equivalent conductor $G$ in the Fig. 5 includes the junction resistor $r_{\mathrm{d}}$ and the load $R$ for the following section.

Moreover, the voltage drop occurs due to these resistors. Then, the experimental results of the next section will be lower than the ideal value of this section.

\section{Experimental Verification}

This section experimentally verifies the effectiveness of the proposed resonant $\mathrm{CW}$ circuit. The prototype adopts the proposed 5-stage resonant CW circuit. The experimental conditions are listed in Table 1. For comparison about the experimental output voltage $V_{\text {outexp }}$, the parallel capacitor $C_{\mathrm{r}}$ and the load $R$ are changed. Note that the inductor $L$ in this paper was made by authors and clear rating cannot be determined.

3.1 Waveforms Fig.7 illustrates the experimental waveforms of the input voltage $v_{\text {in }}(\mathrm{CH} 1)$, the inductor voltage $v_{\mathrm{L}}(\mathrm{CH} 3)$, and the input current $i_{\text {in }}(\mathrm{CH} 2)$ at the resonant frequency of the proposed resonant $\mathrm{CW}$ circuit with $C_{\mathrm{r}}=100 \mathrm{pF}$. Other conditions are presented in Table 1. The inductor voltage $v_{\mathrm{L}}$ is boosted and is much larger than the input voltage $v_{\text {in }}$ thanks to the $L C$ resonance. It is same characteristics as Fig. 3. On the other hand, the inductor voltage $v_{\mathrm{L}}$ in Fig. 7 is smaller than the one in Fig. 3 because the simulation results in Fig. 3 has no internal resistor $r_{\mathrm{L}}$ of the inductor $L$.

Although the input voltage source $v_{\text {in }}$ in Fig. 3 is ideal square wave, the one in Fig. 7 uses a bipolar power supply. Therefore, the input voltage $v_{\text {in }}(\mathrm{CH} 1)$ has the oscillation when the input voltage $v_{\text {in }}$ changes between positive and negative. At this moment, the inductor voltage $v_{\mathrm{L}}(\mathrm{CH} 3)$ shifts several voltages by the input voltage $v_{\text {in }}(\mathrm{CH} 1)$.

\footnotetext{
Some parameters; $C_{\mathrm{t}}, C_{\mathrm{r}}, C, r_{\mathrm{d}}, r_{\mathrm{L}}$, and $L$ depend on frequency and voltage. Therefore, these are not uniquely determined. Section 3 estimates the parameters and calculate $Q$ and $F$.
}

Table 1. Experimental conditions

\begin{tabular}{l|c|l}
\hline \hline Input voltage & $v_{\text {in }}$ & $10 \mathrm{~V}_{\mathrm{amp}}$, square-wave \\
Inductor & $L$ & $127 \mu \mathrm{H}, 1.2 \Omega$ \\
Capacitors & $C$ & $8200 \mathrm{pF}, 1600 \mathrm{~V}$ \\
Fast-recovery diodes & $D_{1}, D_{2}, \ldots, D_{10}$ & $(\mathrm{MUR} 460,600 \mathrm{~V}, 4 \mathrm{~A})$ \\
Parallel capacitor & $C_{\mathrm{r}}$ & $100 \mathrm{pF}, 15 \mathrm{kV}$ \\
& & $1000 \mathrm{pF}, 15 \mathrm{kV}$ \\
\hline
\end{tabular}

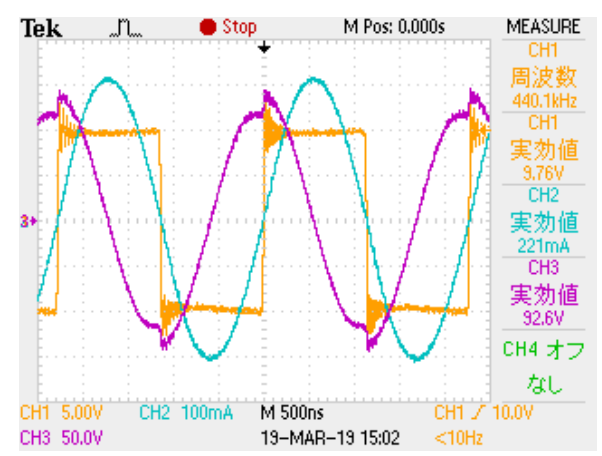

Fig. 7. Experimental input voltage $(\mathrm{CH} 1)$, inductor voltage $(\mathrm{CH} 3)$, and input current $(\mathrm{CH} 2)$ waveforms at resonant frequency with $C_{\mathrm{r}}=100 \mathrm{pF}$

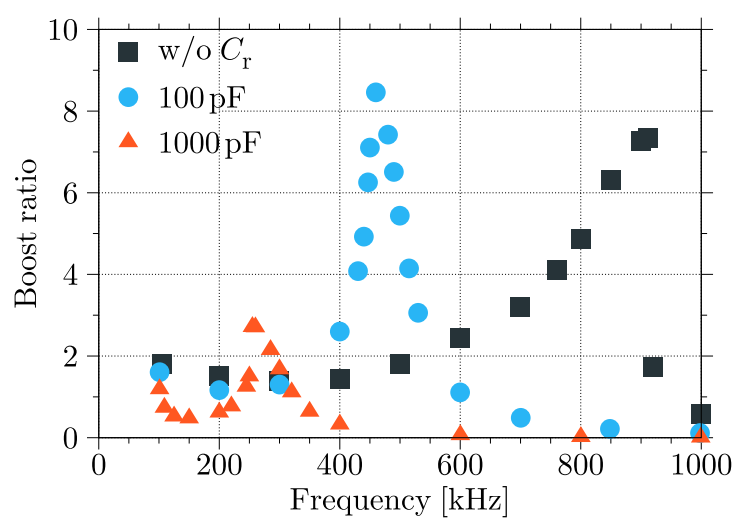

Fig. 8. Frequency characteristics of output voltage at each parallel capacitor

3.2 Frequency Characteristics of Output Voltage Under Various Parallel Capacitors Fig. 8 depicts the experimental frequency characteristics of thew boost ratio, which is defined as the ratio of the output voltage $V_{\text {out,exp }}$ of the proposed resonant $\mathrm{CW}$ circuit to the ideal output voltage $100 \mathrm{~V}$ of the conventional $\mathrm{CW}$ circuit, under various parallel capacitors $C_{\mathrm{r}}$. Other conditions are presented in Table 1. Therefore, a boost ratio greater than 1 means that the output voltage $V_{\text {outexp }}$ of the proposed 5-stage resonant $\mathrm{CW}$ circuit exceeds the ideal and theoretical output voltage of the conventional 5-stage $\mathrm{CW}$ circuit. The keys in Fig. 8 show the capacitance of $C_{\mathrm{r}}$, and "w/o $C_{\mathrm{r}}$ " means no parallel capacitor are inserted.

At first, the result at $C_{\mathrm{r}}=100 \mathrm{pF}$ is focused. The maximum boost ratio, which is achieved at the frequency $460 \mathrm{kHz}$, is about 8.5. The output voltage $V_{\text {out,exp }}$ exceeds the ideal and the theoretical output voltage of the conventional 5-stage $\mathrm{CW}$ circuit. The frequency $460 \mathrm{kHz}$, where the boost ratio becomes the maximum value, is close to the theoretical resonant frequency $424 \mathrm{kHz}$ calculated from (5). Thus, the output voltage $V_{\text {out,exp }}$ is considered to be boosted by the $L C$ resonance between the inductor $L$ and the equivalent capacitor 


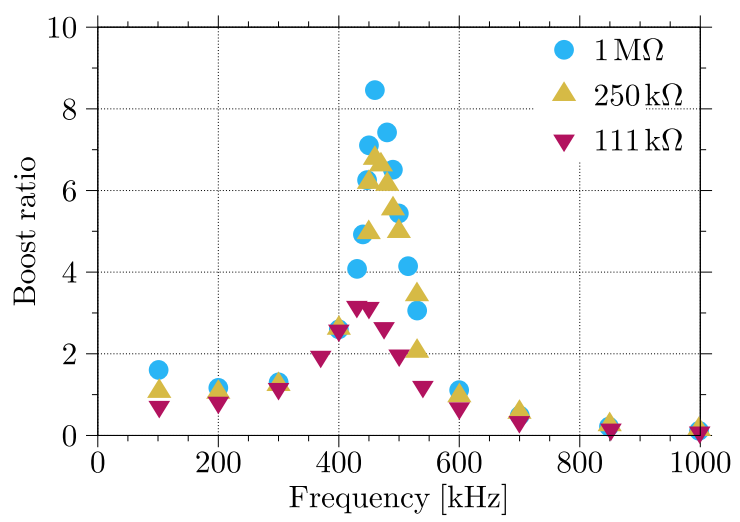

Fig. 9. Frequency characteristics of output voltage under each load

$C_{\text {all }}$.

Secondly, the variations in the boost ratio due to the parallel capacitors $C_{\mathrm{r}}$ is observed. The maximum boost ratio in the case of no parallel capacitors $C_{\mathrm{r}}$ also exceeds 1 as shown in Fig. 8. It is considered that the peak boost ratio generated by the $L C$ resonance between the inductor $L$ and the diode junction capacitors $C_{\mathrm{t}}$. On the other hand, although the maximum boost ratio at the parallel capacitor $C_{\mathrm{r}}=1000 \mathrm{pF}$ exceeds 1 , it is lower than the maximum boost ratio at $C_{\mathrm{r}}=100 \mathrm{pF}$. This is caused by the lower quality factor $Q$ as detailed in Section 4. In addition, relatively low voltage efficiency $F$ also drops the output voltage $V_{\text {out,exp }}$ in the case of the parallel capacitor $C_{\mathrm{r}}=1000 \mathrm{pF}$.

3.3 Frequency Characteristics of Output Voltage Under Various Loads Fig. 9 depicts the experimental frequency characteristics of the boost ratio under the various loads $R$ at the parallel capacitor $C_{\mathrm{r}}=100 \mathrm{pF}$. The keys in Fig. 9 show the load resistor of $R$.

The heavier load $R$ leads into the lower output voltage $V_{\text {out,exp }}$ as shown in Fig. 9. The load current of the proposed resonant $\mathrm{CW}$ circuit becomes large on account of the heavier load. Then, the output voltage $V_{\text {outexp }}$ decreases and the junction capacitor $C_{\mathrm{t}}$ and the junction resistor $r_{\mathrm{d}}$ in the diodes change ${ }^{(18)}$. As a result, the quality factor $Q$ in the proposed resonant $\mathrm{CW}$ circuit changes and the output voltage $V_{\text {out,exp }}$ also changes.

\section{Comparison between Theoretical and Experi- mental Output Voltage}

This section compares the theoretical output voltage $V_{\text {out,the }}$ in Subsection 2.4 and the experimental output voltage $V_{\text {out,exp }}$ in Section 3. In order to calculate the theoretical output voltage $V_{\text {out,the }}$, the equivalent capacitor $C_{\text {all }}$ and the equivalent conductor $G$ have to be calculated first. Since the reverse voltage of the diodes in the proposed resonant $\mathrm{CW}$ circuit changes from zero to $2\left(v_{\text {in }}-v_{\mathrm{L}}\right)$ in the one period, the junction capacitor $C_{\mathrm{t}}$ and the junction resistor $r_{\mathrm{d}}$, which depend on its reverse voltage ${ }^{(18)}$, are changes in the one period. Therefore, the equivalent capacitor $C_{\text {all }}$ and the equivalent conductor $G$ are obtained with the input impedance, which is calculated from the input voltage $v_{\text {in }}$ and the input current $i_{\text {in }}$, in this paper. The first harmonic approximation is used for the calculation of the equivalent capacitor $C_{\text {all }}$ and the equivalent conductor $G^{(23)(24)}$. As a result, the average value of the
Table 2. Calculated quality factor and output voltage under each parallel capacitance at $R=1.0 \mathrm{M} \Omega$

\begin{tabular}{rc|ccc}
\hline \hline Parallel capacitance & $C_{\mathrm{r}}[\mathrm{pF}]$ & $\mathrm{w} / \mathrm{o} C_{\mathrm{r}}$ & 100 & 1000 \\
\hline Quality factor & $Q$ & 6.68 & 8.46 & 5.75 \\
Voltage efficiency & $F$ & 0.977 & 0.891 & 0.534 \\
Theoretical output voltage & $V_{\text {out,the }}[\mathrm{V}]$ & 719 & 823 & 341 \\
Experimental output voltage & $V_{\text {out,exp }}[\mathrm{V}]$ & 734 & 845 & 271 \\
Error & $\varepsilon[\%]$ & 2.0 & 2.6 & 20.5 \\
\hline
\end{tabular}

Table 3. Calculated quality factor and output voltage under each load at $C_{\mathrm{r}}=100 \mathrm{pF}$

\begin{tabular}{rc|ccc}
\hline \hline Load & $R[\mathrm{k} \Omega]$ & 1000 & 250 & 111 \\
\hline Quality factor & $Q$ & 8.46 & 7.33 & 4.10 \\
Voltage efficiency & $F$ & 0.891 & 0.891 & 0.891 \\
Theoretical output voltage & $V_{\text {out,the }}[\mathrm{V}]$ & 823 & 718 & 416 \\
Experimental output voltage & $V_{\text {out,exp }}[\mathrm{V}]$ & 845 & 677 & 316 \\
Error & $\varepsilon[\%]$ & 2.6 & 5.7 & 24.0 \\
\hline
\end{tabular}

equivalent capacitor $C_{\text {all }}$ and the equivalent conductor $G$ in the one period are obtained. In addition, the inductor $L$ and internal resistor of the inductor $r_{\mathrm{L}}$ is measured values. Besides, the diode junction capacitor $C_{\mathrm{t}}$ in (12) is obtained from the equivalent capacitor $C_{\text {all }}$ when no parallel capacitors are inserted, where $C_{\mathrm{t}}=C_{\text {all }} / 2 N$.

Table 2 shows the quality factor $Q$, the voltage efficiency $F$, the theoretical output voltage $V_{\text {out,the }}$, the experimental output voltage $V_{\text {out,exp }}$, and the error $\varepsilon$ under each parallel capacitor at the load resistor $R=1.0 \mathrm{M} \Omega$. The quality factor $Q$ is the largest at the parallel capacitor $C_{\mathrm{r}}=100 \mathrm{pF}$, and the output voltage $V_{\text {out,the }}$ and $V_{\text {out,exp }}$ also become the largest. Hence, the improvement of the quality factor $Q$ contributes

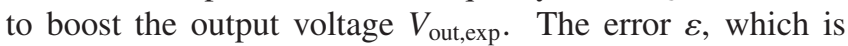
defined as $\varepsilon=\left|V_{\text {out,the }}-V_{\text {out,exp }}\right| / V_{\text {out,exp }}$, is less than $3 \%$ at "w/o $C_{\mathrm{r}}$ " and " $C_{\mathrm{r}}=100 \mathrm{pF}$ " as shown in Table 2. Therefore, (11) is considered to be valid. The error $\varepsilon$ at "w/o $C_{\mathrm{r}}$ " and " $C_{\mathrm{r}}=100 \mathrm{pF}$ " is considered to be caused by the measurement error of the inductor $L$ and the resistor $r_{\mathrm{r}}$. On the other hand, the error $\varepsilon=20.7 \%$ is relatively large at $C_{\mathrm{r}}=1000 \mathrm{pF}$. The voltage drop at the capacitors $C_{1}, C_{2}, \ldots, C_{10}$ cannot be ignored since the parallel capacitors $C_{\mathrm{r}}$ have a value of the capacitor close to the capacitor $C$ at $C_{\mathrm{r}}=1000 \mathrm{pF}$. As a result, the theoretical output voltage $V_{\text {out,the }}$ becomes larger than the experimental output voltage $V_{\text {out,exp }}$.

Table 3 lists the quality factor $Q$, the voltage efficiency $F$, the theoretical output voltage $V_{\text {out,the }}$, the experimental output voltage $V_{\text {out,exp }}$, and error $\varepsilon$ under each load and parallel capacitance $C_{\mathrm{r}}=100 \mathrm{pF}$. The heavier load $R$ leads lower theoretical output voltage $V_{\text {out,the }}$ as well as the experimental output voltage $V_{\text {out,exp }}$. The error $\varepsilon$ becomes larger as the load $R$ becomes heavier because (11) does not consider voltage drop due to the load current.

\section{Conclusion}

This paper derives the theoretical quality factor and the output voltage of the proposed resonant $\mathrm{CW}$ circuit considering the equivalent capacitor and the equivalent resistor. It is clarified that the additional parallel capacitors improve the quality factor and the output voltage of the proposed resonant CW circuit. Finally, the experimental results reveal the validity of the theoretical analysis.

This paper does not clarify a specific design procedure of 
the inductor and the parallel capacitors because of nonlinear characteristics of the equivalent capacitor and the equivalent conductor in the $\mathrm{CW}$ circuit. In future work, a specific design procedure of the inductor and the parallel capacitors will be considered in this research.

\section{Acknowledgment}

The authors would like to appreciate Prof. M. Ishitobi and members of High-Frequency Power Applications Laboratory in National Institute of Technology, Nara College for their support.

\section{References}

( 1 ) J.D. Cockcroft and E.T. Walton: "Experiments with High Velocity Positive Ions.-(I) Further Developments in the Method of Obtaining High Velocity Positive Ions", Proceedings of the Royal Society of London. Series A, Vol.136, No.830, pp.619-630 (1932)

( 2 ) M.D. Bellar, E. Watanabe, and A. Mesquita: "Analysis of the Dynamic and Steady-State Performance of Cockcroft-Walton Cascade Rectifiers", IEEE Transactions on Power Electronics, Vol.7, No.3, pp.526-534 (1992)

( 3 ) S. Mao, J. Popović, and J.A. Ferreira: "Diode Reverse Recovery Process and Reduction of a Half-Wave Series Cockcroft-Walton Voltage Multiplier for High-Frequency High-Voltage Generator Applications", IEEE Transactions on Power Electronics, Vol.34, No.2, pp.1492-1499 (2019)

( 4 ) J. Brugler: "Theoretical Performance of Voltage Multiplier Circuit", IEEE Journal of Solid-State Circuits, Vol.6, No.3, pp.132-135 (1971)

( 5 ) E. Everhart and P. Lorrain: "The Cockcroft-Walton Voltage Multiplying Circuit", Review of Scientific Instruments, Vol.24, No.3, pp.221-226 (1953)

( 6 ) M. Minami, T. Ito, S. Motegi, and M. Michihira: "Theoretical Analysis of Decreased Boost Ratio in Unloaded Cockcroft-Walton Circuit (in Japanese)", IEEJ Transactions on Industry Applications, Vol.136, No.3, pp.246-247 (2016)

( 7 ) S. Iqbal: "A Three-Phase Symmetrical Multistage Voltage Multiplier", IEEE Power Electronics Letters, Vol.3, No.1, pp.30-33 (2005)

( 8 ) I.C. Kobougias and E.C. Tatakis: "Optimal Design of a Half-Wave Cockcroft-Walton Voltage Multiplier with Minimum Total Capacitance", IEEE Transactions on Power Electronics, Vol.25, No.9, pp.2460-2468 (2010)

(9) J. Biela and J.W. Kolar: "Using Transformer Parasitics for Resonant Converters-A Review of the Calculation of the Stray Capacitance of Transformers", IEEE Transactions on Industry Applications, Vol.44, No.1, pp.223-233 (2008)

(10) N. Shafiei, M. Pahlevaninezhad, H. Farzanehfard, A. Bakhshai, and P. Jain: "Analysis of a Fifth-Order Resonant Converter for High-Voltage DC Power Supplies", IEEE Transactions on Power Electronics, Vol.28, No.1, pp.85100 (2013)

(11) B. Kang, K.-S. Low, J.J. Soon, and Q.-V. Tran: "Single-Switch QuasiResonant DC-DC Converter for a Pulsed Plasma Thruster of Satellites", IEEE Transactions on Power Electronics, Vol.32, No.6, pp.4503-4513 (2017)

(12) S.D. Johnson, A.F. Witulski, and R.W. Erickson: "A Comparison of Resonant Topologies in High Voltage DC Applications", in 1987 2nd IEEE Applied Power Electronics Conference and Exposition, pp.145-156 (1987)

(13) J. Biela, M. Schweizer, S. Waffler, and J.W. Kolar: "SiC versus Si-Evaluation of Potentials for Performance Improvement of Inverter and DC-DC Converter Systems by SiC Power Semiconductors", IEEE Transactions on Industrial Electronics, Vol.58, No.7, pp.2872-2882 (2011)

(14) Y. Du, J. Wang, G. Wang, and A.Q. Huang: "Modeling of the HighFrequency Rectifier with $10 \mathrm{kV}$ SiC JBS Diodes in High-Voltage Series Resonant Type DC-DC Converters", IEEE Transactions on Power Electronics, Vol.29, No.8, pp.4288-4300 (2014)

(15) L. Katzir and D. Shmilovitz: "A 1-MHz 5-kV Power Supply Applying SiC Diodes and GaN HEMT Cascode MOSFETs in Soft Switching", IEEE Journal of Emerging and Selected Topics in Power Electronics, Vol.4, No.4, pp.1474-1482 (2016)

(16) M. Minami, T. Ito, S. Motegi, and M. Michihira: "Boost Ratio and Power Factor Improvement in Cockcroft-Walton Circuit with Diode Junction Capacitor (in Japanese)", IEEJ Transactions on Industry Applications, Vol.136, No.12, pp.991-996 (2016)
(17) M. Minami, T. Ito, S. Motegi, and M. Michihira: "An Experimental Analysis of Output Voltage Characteristics in Cockcroft-Walton Circuit with $\mathrm{SiC}$ Diode and Input Inductor", Proceedings of 2016 19th International Conference on Electrical Machines and Systems (ICEMS). IEEE, pp.1-4 (2016)

(18) B.L. Anderson and R.L. Anderson: Fundamentals of Semiconductor Devices. McGraw-Hill, Inc. (2004)

(19) R.L. Steigerwald: "A Comparison of Half-Bridge Resonant Converter Topologies", IEEE Transactions on Power Electronics, Vol.3, No.2, pp.174$182(1988)$

(20) G. Ivensky, A. Kats, and S. Ben-Yaakov: "An RC Load Model of Parallel and Series-Parallel Resonant DC-DC Converters with Capacitive Output Filter", IEEE Transactions on Power Electronics, Vol.14, No.3, pp.515-521 (1999)

(21) C.M. Young, M.H. Chen, T.A. Chang, C.C. Ko, and K.K. Jen: "Cascade Cockcroft-Walton Voltage Multiplier Applied to Transformerless High StepUp DC-DC Converter", IEEE Transactions on Industrial Electronics, Vol.60, No.2, pp.523-537 (2013)

(22) T. Ohira: "Rigorous Q-Factor Formulation for One- and Two-Port Passive Linear Networks from an Oscillator Noise Spectrum Viewpoint", IEEE Transactions on Circuits and Systems II: Express Briefs, Vol.52, No.12, pp.846-850 (2005)

(23) R. Beiranvand, B. Rashidian, M.R. Zolghadri, and S.M.H. Alavi: "A Design Procedure for Optimizing the LLC Resonant Converter as a Wide Output Range Voltage Source", IEEE Transactions on Power Electronics, Vol.27, No.8, pp.3749-3763 (2012)

(24) W.C. Hsu, J.F. Chen, Y.P. Hsieh, and Y.M. Wu: "Design and Steady-State Analysis of Parallel Resonant DC-DC Converter for High-Voltage Power Generator", IEEE Transactions on Power Electronics, Vol.32, No.2, pp.957966 (2017)

Masataka Minami (Member) received the Bachelor's, Master's, and

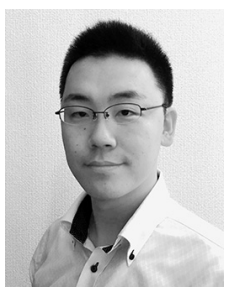
Ph.D. degrees from Kyoto University, Kyoto, Japan, in 2008, 2010, and 2013, respectively. In 2013, he joined the Department of Electrical Engineering, Kobe City College of Technology (KCCT), where he is currently an Associate Professor. From April 2018 to March 2019, he was an Academic Visitor in the School of Engineering, Ecole Polytechnique Federale de Lausanne (EPFL), Lausanne, Switzerland. His research interests include rectifiers, DC/DC converters, inverters, power systems engineering, and control applications. He is a member of the Institute of Electronics, Information and Communication Engineers (IEICE) and Institute of System, Control and Information Engineers (ISCIE).

Takumi Yasuda (Student Member) received the Bachelor's degrees

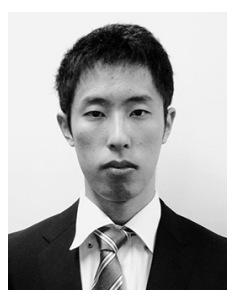
rom Kobe City College of Technology (KCCT) in 2019. Since 2019, he is a Ph.D. candidate in Nagaoka University of Technology.

Jun-ichi Itoh (Senior Member) received his M.S. and Ph.D. degree

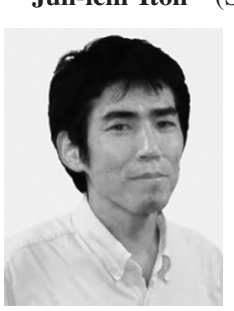
in electrical and electronic systems engineering from Nagaoka University of Technology, Niigata, Japan in 1996, 2000, respectively. From 1996 to 2004, he was with Fuji Electric Corporate Research and Development Ltd., Tokyo, Japan. He was with Nagaoka University of Technology, Niigata, Japan as an associate professor. Since 2017, he has been a professor. His research interests are matrix converters, $\mathrm{dc} / \mathrm{dc}$ converters, power factor correction techniques, energy storage system and adjustable speed drive systems. He is a senior member of the Society of Automotive Engineers of Japan and the IEEE. 